\title{
Total Acetylcholine Content, and Activities of Choline Acetyltransferase and Acetylcholinesterase in Brain and Duodenum of SART-Stressed (Repeated Cold-Stressed) Rat
}

\author{
Taeko HATA, Tomitaro KITA, Takashi HIGASHIGUCHI \\ and Seiji ICHIDA* \\ Department of Pharmacology and *Biological Chemistry. Faculty of Pharmacy, \\ Kinki University. Higashi-Osaka 577. Japan \\ Accepted April 25, 1986
}

\begin{abstract}
The cholinergic activities in SART (specific alternation of rhythm in temperature)-stressed (repeated cold-stressed) rats, which are diseased rats with vagotonic-type dysautonomia, were examined with the following results. 1) $A$ decreased content of total acetylcholine ( $T-A C h$ ) and increased activities of choline acetyltransferase (CAT) and acetylcholinesterase (AChE) in the basal ganglia and an increase in the T-ACh content and decrease in the AChE activity in the duodenum of SART-stressed rats reached the respective plateaus on day 5 of stress, which were maintained thereafter. CAT activity. however, in the hypothalamus was activated most on day 2. 2) These changes in SART-stressed rats were different from those in simple cold-stressed rats. 3) Subdiaphragmatic vagotomy inhibited the appearance of the changes in the duodenum, but not those in the hypothalamus of SART-stressed rats. 4) The sedative analgesic Neurotropin ${ }^{\text {R }}$ prevented all the changes in SART-stressed rats described above. These results suggest that cholinergic neurons may be activated in both the hypothalamus and basal ganglia of the brain of SART-stressed rats, and the characteristic peripheral changes of the cholinergic system in the duodenum of SART-stressed rats may be under the control of the parasympathetic center.
\end{abstract}

The fact that various physiological changes are observed in animals exposed to stress has been systematized by Selye, Cannon and others. Neurohormonal changes caused by stress, especially movements of brain catecholamines, have long been of interest, and there are many reports of the relation of stress with the contents and turnover rates of norepinephrine, dopamine, serotonin, etc. (1-4). However, little is known about the relations between stress and acetylcholine (ACh). There is some evidence that acute stress can alter the ACh content of the central nervous system (CNS). but the results reported are not entirely consistent. Some investigators have shown that stress causes changes in the ACh activity in the CNS (5), increased ACh release, down-regulation of muscarinic re- ceptors and increased choline uptake in stress-sensitive rats (6). Other investigators have reported an increase in the $\mathrm{ACh}$ content and a decrease in $\mathrm{ACh}$ turnover in the frontal cortex of rats after acute stress for $1 \mathrm{hr}$ (7) and an increase in $\mathrm{ACh}$ receptor sites and an increase in ACh turnover in the hypothalamus after stress for 1.4 or $24 \mathrm{hr}(8)$. Moreover, stress or a conditioned stimulus were reported to increase the $\mathrm{ACh}$ level in the CNS (9).

Previously, we reported (10) that a special. repeated cold stress, which we named SART (specific alternation of rhythm in temperature) stress, caused strong stress in mice and rats. We used animals subjected to SART stress in many experiments concerning physiological changes.

SART-stressed animals are pathologically 
diseased animals with vagotonic-type dysautonomia (11), judging from the results of some balance tests on the autonomic nervous system (the mecholyl test. Aschner's oculocardiac test and galvanic skin response test) and they show continued decrease in blood pressure (12). It is especially interesting that these animals are hyperalgesic and show a clear decrease in pain threshold (13) in contrast to animals with common stresses which show so-called stress-induced analgesia. Isolated duodenum from SART stressed animals shows subsensitivity to ACh (14) and a quantitative decrease of muscarinic ACh receptors (15). The decrease in the $\mathrm{ACh}$ response in the isolated duodenum was inhibited by bilateral subdiaphragmatic vagotomy. but not by splanchnic sympathectomy (16). From these facts, SARTstressed animals are thought to be in a partial vagotonic state, at least with respect to their duodenum. Their subsensitivity to $\mathrm{ACh}$ is thought to be caused partly by local independency of parasympathetic nerve activity from the center, but mostly caused by the functional change in their higher parasympathetic center. Then, we have reported changes in the total ACh (T-ACh) content and the activities of choline acetyltransferase (CAT) and acetylcholinesterase (AChE) in the brain and duodenum of SART-stressed rats (17).

In the present study, therefore, we examined in more detail the central and peripheral cholinergic neuron activities of SARTstressed rats. In addition, some rats were exposed to simple cold-stress or restraint plus water-immersion stress (RWIS) for comparison with SART-stressed rats. RWIS is an acute stress of a partially sympathicotonic type $(18,19)$ in contrast to SART stress.

Results suggest that SART stress is closely related with cholinergic neuron activities.

\section{Materiais and Methods}

Male Wistar rats weighing 200-300 g were used. The animals were kept in a temperature-controlled room $\left(24 \pm 1^{\circ} \mathrm{C}\right)$ with a $12 \mathrm{hr}$ light/12 $\mathrm{hr}$ dark cycle (light on between 7 a.m. and 7 p.m.) and received a standard diet and water ad libitum.
As a drug, a dried extract of inflamed skin of rabbits inoculated with vaccinia virus (Neurotropin ${ }^{3}$. NSP for short, Nippon Zoki) was used. NSP was dissolved in physiological saline and injected i.p. into rats at doses of 50 and $100 \mathrm{mg} / \mathrm{kg}$. once daily for 5 days during SART stress, and experiments were carried out on the following day after a final treatment with NSP. In experiments on RWIS rats, rats were pretreated with NSP once daily from 4 days before stress, and they were subjected to RWIS $1 \mathrm{hr}$ after the final treatment with NSP.

SART stress: Rats were kept alternately at $24^{\circ} \mathrm{C}$ and $-3^{\circ} \mathrm{C}$ for $1 \mathrm{hr}$ periods from 9 a.m. to 4 p.m. and then at $-3^{\circ} \mathrm{C}$ from 4 p.m. to 9 a.m. the following morning. This treatment was continued for 5 consecutive days ( 10 . 20).

Cold stress: Rats were kept at $-3^{\circ} \mathrm{C}$ continuously for 2 days.

RWIS: Rats were fastened to a board by their extremities, and they were immersed up to their xiphoid process in water at $25^{\circ} \mathrm{C}$ for $3 \mathrm{hr}$ (18).

Determination of T-ACh content: For extraction of brain $A C h$, rats were sacrificed by overhead irradiation with $2,450 \mathrm{MHz}$ microwaves at $5 \mathrm{~kW}$ for $1.2 \mathrm{sec}$ from a microwave applicator (Toshiba, TMW$6402 \mathrm{~A}$ ) to inactivate enzymes involved in $\mathrm{ACh}$ synthesis and metabolism. They were then decapitated, and their brain was excised and the cerebral cortex, basal ganglia, hypothalamus and hippocampus were separated by the method of Gispen et al. (21). ACh was extracted from these regions by the formic acid-acetone technique of Toru and Aprison (22). For extraction of duodenal ACh, rats were killed by decapitation. and a length of $25 \mathrm{~mm}$ of duodenum was isolated, immersed in Tyrode's solution $(\mathrm{pH}$ 4.0) containing physostigmine, homogenized in a homogenizer (Niti-On. NS-501, Physcotron), and allowed to stand in icewater for $30 \mathrm{~min}$ to extract ACh, by the method of Yagasaki et al. (23).

The T-ACh contents of samples obtained from the brain and duodenum were determined by bioassay with isolated guinea-pig ileum and an isotonic transducer (Nihon Kohden, TD-112S), by the method of Blaber 
and Cuthbert (24). The medium used in this case was the Tyrode's solution containing physostigmine sulphate (Sigma, $5 \times 10^{-8} \mathrm{M}$ ) as an AChE-inhibitor, tryptamine hydrochloride (Wako, $2 \times 10^{-4} \mathrm{M}$ ) as an antiserotonin drug. and mepyramine maleate (Sigma, $2.5 \times 10^{-6} \mathrm{M}$ ) as an anti-histamine drug. $\alpha$-Chymotrypsin (Sigma, $400 / / \mathrm{g} / \mathrm{ml}$ ) which shows anti-substance $P$ activity and amberlite XAD-2 (Rohm and Haas, $0.1 \mathrm{ml}$ / $\mathrm{ml}$ ) with anti-prostaglandin activity were added to the sample solution immediately before the bioassay and incubated with the preparation at $38^{\circ} \mathrm{C}$ for $10 \mathrm{~min}$.

Assay of CAT activity: Rats were killed by decapitation, the brain was excised and separated into various areas on crushed ice by the method of Gispen et al. (21), and a length of $25 \mathrm{~mm}$ of duodenum was isolated. The samples obtained from the brain and duodenum were weighed and put into icecold phosphate buffer solution ( $\mathrm{pH} 7.0$. $0.01 \mathrm{M}, 10$ volumes of samples) containing EDTA.2Na.2 $\mathrm{H}_{2} \mathrm{O}(1 \mathrm{mM})$ and Triton $\mathrm{X}-100$ $(0.1 \%)$.

CAT activity was assayed by the radiochemical method of Fonnum (25). A solution containing EDTA.2 $\mathrm{Na} \cdot 2 \mathrm{H}_{2} \mathrm{O}, \quad \mathrm{Na}_{2} \mathrm{HPO}_{4}$. $12 \mathrm{H}_{2} \mathrm{O}$. $\mathrm{NaCl}$, bovine serum albumin, choline chloride and physostigmine sulphate was prepared. The test solution was mixed with a half volume of this solution and the same volume of ${ }^{3} \mathrm{H}$-labeled acetylcoenzyme $\mathrm{A}$ (Amersham, $0.55 \mathrm{Ci} / \mathrm{mmol} ; 0.4 \mathrm{mM}, 12.5$ $\mu \mathrm{Ci} / \mathrm{ml}$ ) and incubated in a water-bath at $37^{\circ} \mathrm{C}$ for 20 min to allow synthesis of $3 \mathrm{H}$ $\mathrm{ACh}$. The reaction was stopped by adding phosphate buffer (pH 7.4, $0.01 \mathrm{M}$ ) containing $\mathrm{ACh}$ chloride (Daiichi, Ovisot ${ }^{(\mathbb{B})}$ ), and ${ }^{3} \mathrm{H}$-ACh was extracted into an organic solvent consisting of butyl ethyl ketone (Aldrich, 3Heptanon), diethylether (Wako) and sodium tetraphenylboron (Nakarai). Then the radioactivity of ${ }^{3} \mathrm{H}-\mathrm{ACh}$ was counted in a liquid scintillation counter (Packard, Liquid Scintillation Spectrometer TR1-CARB 3380). CAT activity was calculated from the known activity of ${ }^{3} \mathrm{H}$-acetyl-coenzyme $\mathrm{A}$.

Assay of AChE activity: Brain and duodenum tissues were isolated as described in the CAT assay: they were homogenized in $0.1 \mathrm{M}$-phosphate buffer ( $\mathrm{pH} 8.0)$, and the supernatants of the resulting homogenates were used as samples. AChE activity was determined by the photometric method of Ellman et al. (26) by following the increase of absorbance at $412 \mathrm{~nm}$ of the yellow compound (5-thio-2-nitrobenzoic acid) formed from the reaction product with acetylthiocholine iodide as the substrate and $5,5^{\circ}$ dithiobis-2-nitrobenzoic acid. Absorbance was measured in a spectrophotometer (Hitachi, 100-10).

Vagotomy: Rats were anesthetized with sodium pentobarbital (Abbott, Nembutal ${ }^{\circledR}$ ). and their vagus nerves were cut bilaterally $1 \mathrm{~cm}$ above the cardia under a stereoscopic microscope $(\times 8)$ by the method of Toriumi et al. (27).

Statistical analysis: All data obtained are given as the mean $\pm S$.E. The significance of differences was examined by Student's $t$ test.

\section{Results}

1. Time courses of changes in the $A C h$ content and CAT and AChE activities in the brain and duodenum of SART-stressed rats: Figure 1 shows the time courses of changes in the T-ACh content in the brain and duodenum of rats under continuous SART stress. The T-ACh contents of the basal ganglia and hypothalamus started to decrease from day 1 (from about $6 \mathrm{hr}$ ) of SART stress. The decreases were significant $(P<0.01)$ after 3 days, and the decreased levels were maintained during an additional three weeks of stress. In contrast, in the duodenum, the $\mathrm{T}$-ACh content increased from day 1 of SART stress, and the increase was significant after 3 days and reached a maximum after 5 days, which was subsequently maintained. The T-ACh content of the cerebral cortex did not change.

Figure 2 shows the time courses of changes in the CAT activities in the brain and duodenum. The hypothalamus showed a significant increase in CAT activity on day 1 (after $6 \mathrm{hr}$ ) of stress. The activity was maximal after $24 \mathrm{hr}$, but remained at a high level for more than 2 weeks. In the basal ganglia, the CAT activity increased gradually, to a maximum after 5 days, but returned to the normal level after 2 weeks. The thalamus, 


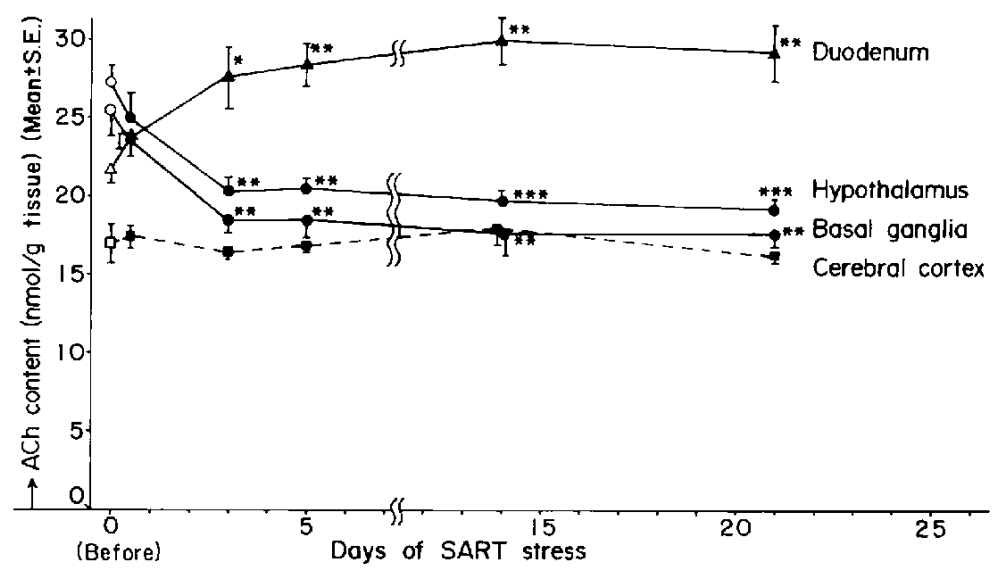

Fig. 1. Changes of $\mathrm{ACh}$ contents in rat brain areas and duodenum during SART stress. Values are means \pm S.E. for $4-7$ rats. $\quad P<0.05,{ }^{*} P<0.01$, ${ }^{* *} P<0.001$ : Significance of difference from the respective value before stress by Student's $t$-test.

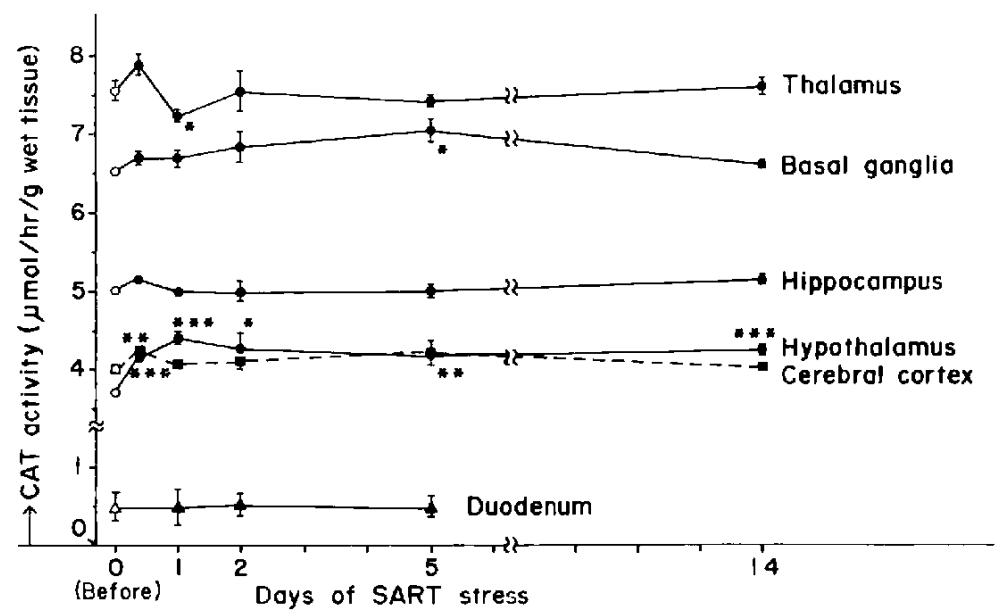

Fig. 2. Changes of CAT activities in rat brain areas and cuodenum during SART stress. Values are means \pm S.E. for $5-7$ rats. $" \mathrm{P} \times 0.05 .{ }^{*} \mathrm{P}<0.01 .{ }^{* * P}<0.001$ : Significance of difference from the respective value before stress by Student's $t$-test.

hippocampus and cortex showed no change in activity during the 2 weeks of stress. The duodenum also showed no change in activity.

Figure 3 shows the time courses of changes in AChE activity during stress. The AChE activity in the basal ganglia increased significantly after one day and reached a maximum on day 5 , which was maintained during the 2 weeks of stress. The activity in the hypothalamus increased significantly after 2 days and reached a plateau on day 3 , which was maintained thereafter. The activity in the duodenum did not change appreciably for 3 days, but then decreased markedly and remained at a low level from day 5 .

2. Recovery from changes in ACh content and CAT and AChE activities after cessation of SART stress: Figure 4 shows the time courses of changes in ACh contents and enzyme activities after the end of SART stress. The T-ACh content of the duodenum was still high 14 days after stress termination. but returned to the normal level after 21 days. 


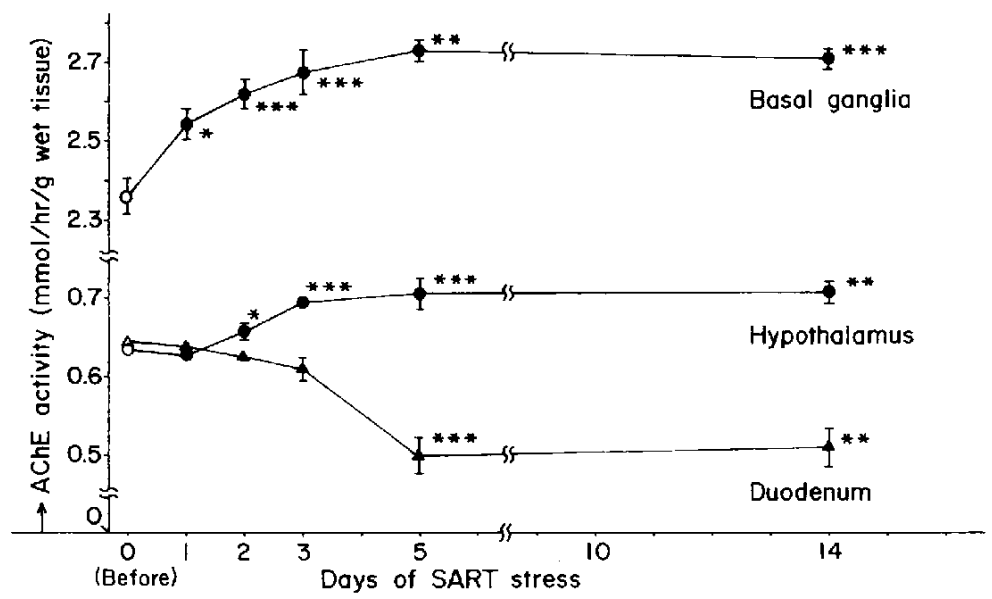

Fig. 3. Changes of AChE activities in rat brain areas and duodenum during SART stress. Values are means $\pm S$. E. for $4-7$ rats. ${ }^{*} P<0.05,{ }^{*} P<0.01 .{ }^{* * P} P<0.001$ : Significance of difference from the respective value before stress by Student's $t$-test.

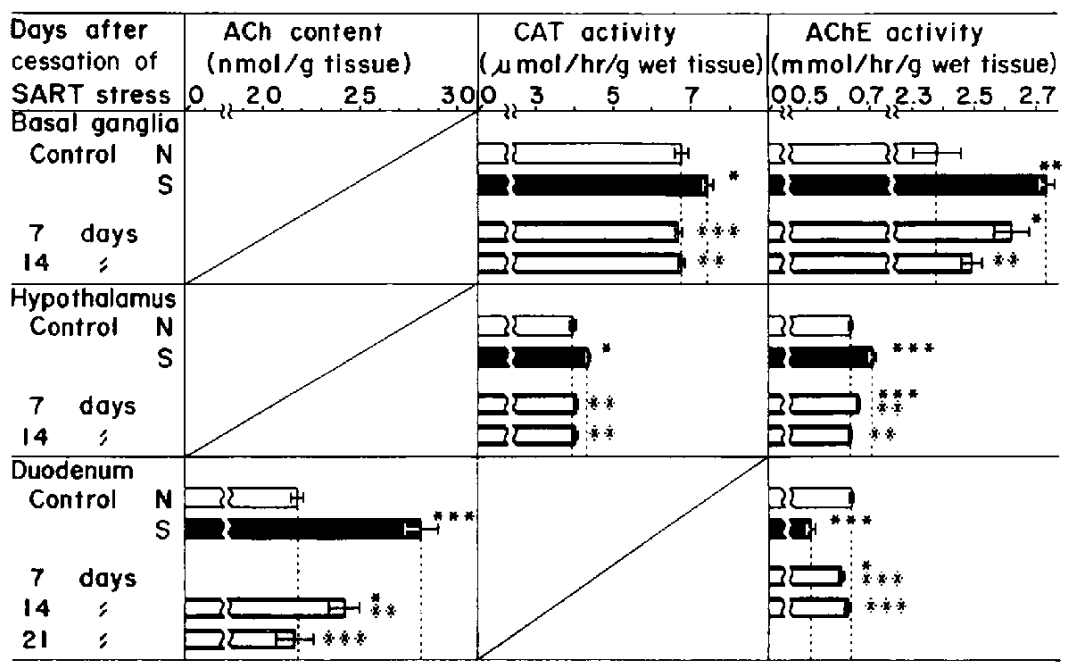

Fig. 4. ACh content, and CAT and AChE activities in rat brain areas and duodenum after cessation of SART stress. $N$ : no stress; S: SART stress. Values are means $\pm S . E$. for $4-7$ rats. ${ }^{*} P<0.05,{ }^{* *} P<0.01$. ${ }^{* *} P<0.001$ : Significance of difference from the value for the non-stressed controls. $\% \mathrm{P}<0.01$. \% $\%$ < 0.001 : Significance of difference from SART-stressed controls (Student's $t$-test).

On the other hand, the CAT activities in the basal ganglia and hypothalamus returned to the normal levels within only 7 days after the end of stress. The AChE activity in the basal ganglia remained at a significantly increased level for 7 days, but returned to the control level after 14 days. The activities in the hypothalamus and duodenum returned to normal levels after 7 days.
3. Comparison of changes in cholinergic characteristics of the brain and duodenum of SART- and cold-stressed rats: Figure 5 shows the T-ACh contents and CAT and AChE activities in the brain and duodenum of SART- and cold-stressed rats as percentages of the mean values in normal rats. The T-ACh contents of the basal ganglia and hypothalamus of cold-stressed rats showed sig- 


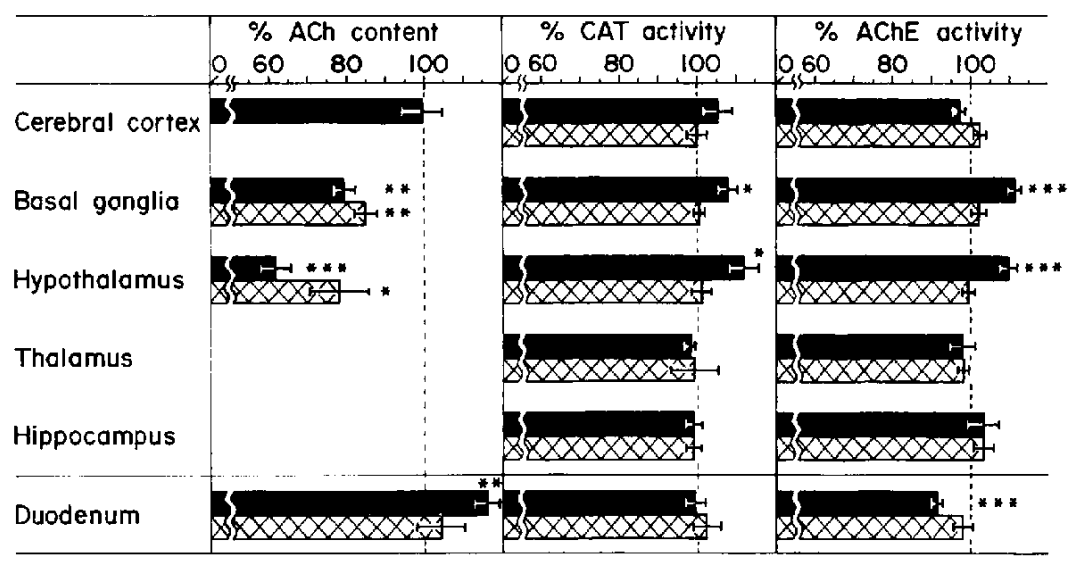

Fig. 5. Comparison of changes in cholinergic characteristics of SART- and cold-stressed rat brain areas and duodenum. SART stress, $|\times \bar{x}|$ cold stress. Values are percentages of those for unstressed rats, and they are mearis $\pm S . E$. for $4-10$ rats. ${ }^{*} P<0.05,{ }^{* *} P<0.01,{ }^{* *} P<0.001$ : Significance of difference from the value in unstressed rats (Student's $t$-test).

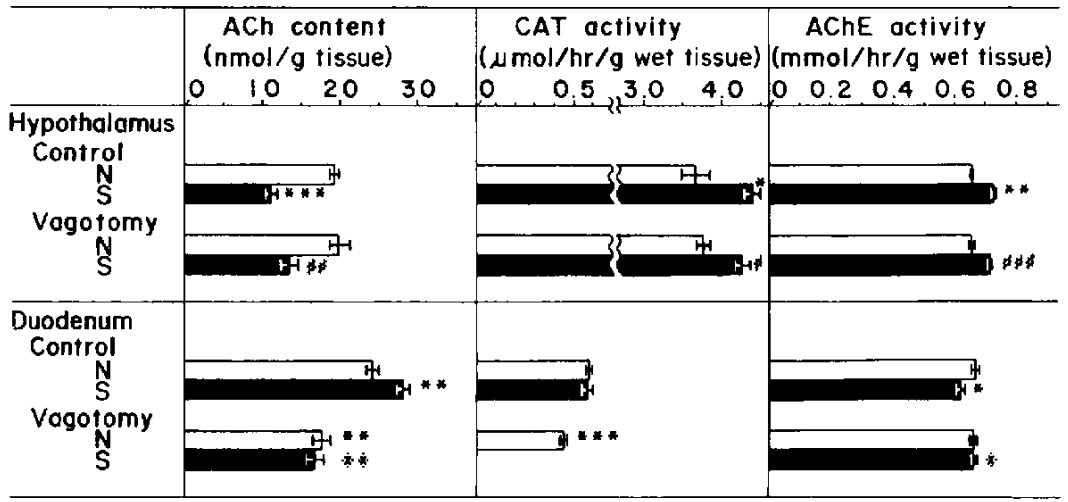

Fig. 6. ACh content, and CAT and AChE activities in the hypothalamus and duodenum of vagotomized plus SART-stressed rats. $N$ : non-stress, S: SART-stress. Values are means $\pm S . E$. for $4-8$ rats. ${ }^{*} P<0.05$. ${ }^{*} P<0.01,{ }^{* *} P<0.001$ : Significance of difference from the value for the non-stressed controls. $\% P<0.05$. $\therefore \leftrightarrow P<0.01$ : Significance of difference from the value for SART-stressed controls. $\because P<0.05, \$ P<0.01$. *t: $\mathrm{P}<0.001$ : Significance of difference from the value for the respective non-stressed group (Student's t-test).

nificant decreases, although less than those in SART-stressed rats. The CAT and AChE activities did not change in cold-stressed rats, in contrast to those in SART-stressed rats.

Thus, unlike SART-stressed rats, coldstressed rats showed only a slight decrease in T-ACh content.

RWIS rats showed only an increase in A.ChE activity in the duodenum (data not shown).

4. Changes in cholinergic characteristics in the hypothalamus and duodenum of vagotomized plus SART-stressed rats: Figure 6 shows data on the T-ACh contents, and the CAT and AChE activities in unstressed vagotomized rats and rats exposed to SART stress after vagotomy. Vagotomy did not influence any of the three parameters in the hypothalamus of unstressed rats. Moreover, the decrease in the T-ACh content and increases in the CAT and AChE activities in the hypothalamus were similar in SART- 


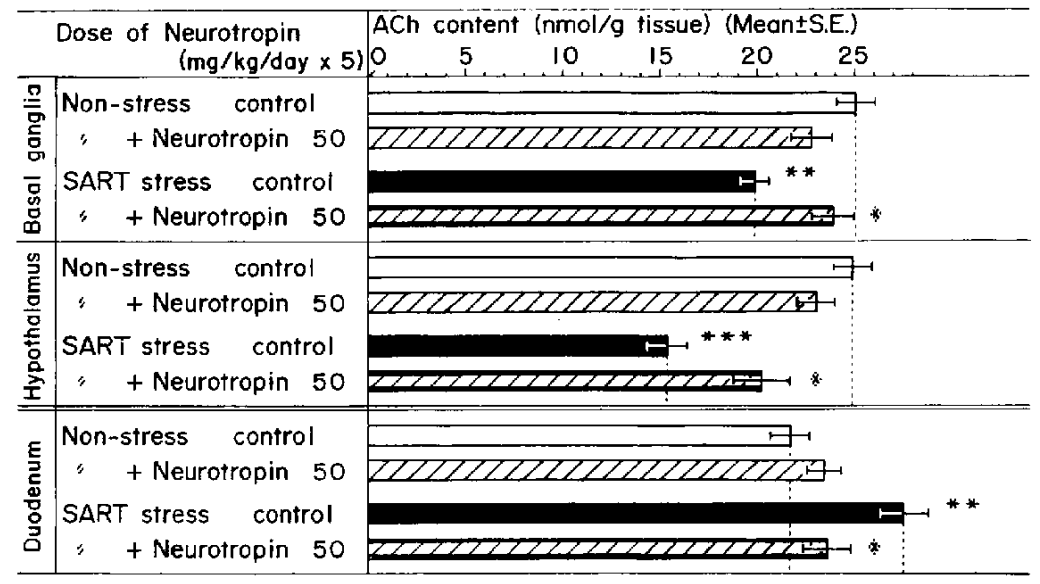

Fig. 7. Effects of Neurotropin on changes of ACh contents of the brain areas and duodenum of SARTstressed rats. No. of rats: $4-10 /$ group. ${ }^{* *} P<0.01,{ }^{* *} P<0.001$ : Significance of difference from the value of the respective non-stressed groups. $\$ P<0.05$ : Significance of difference from the value for SART-stressed controls (Student's $t$-test).

stressed rats with and without vagotomy. Vagotomy induced decrease in the T-ACh content and CAT activity, but caused no change in the AChE activity in the duodenum of unstressed rats. In the duodenum of rats after vagotomy plus SART stress, the T-ACh content and AChE activity were much the same as those after vagotomy without stress. Thus the increase in the duodenal T-ACh content and decrease in the AChE activity induced by SART stress were inhibited by vagotomy.

The duodenal CAT activity of vagotomized plus SART-stressed rats was not measured because no change was observed in this activity in SART-stressed rats.

5. Effects of NSP on changes in cholinergic parameters in the brain and duodenum of SART-stressed rats and the duodenum of RWIS-loaded rats: Figure 7 shows the effect of NSP on changes in the T-ACh contents of SART-stressed rats. In unstressed rats, NSP at a dose of $50 \mathrm{mg} / \mathrm{kg} /$ day $\times 5$ had no influence on the $\mathrm{T}$-ACh content in the basal ganglia, hypothalamus or duodenum. In SART-stressed rats, it significantly inhibited the decrease in the T-ACh content in the basal ganglia and hypothalamus and inhibited the increase in the T-ACh content in the duodenum, the protecting effects being $78.4,50.5$ and $67.3 \%$, respectively.
The increase in the $\mathrm{T}$-ACh content in mouse duodenum was also significantly inhibited by five successive treatments with NSP at a dose of $100 \mathrm{mg} / \mathrm{kg} /$ day (data not shown).

Figure 8 shows the effect of NSP on changes in CAT and AChE activities in SARTstressed rats. Five successive treatments with NSP at a dose of $50 \mathrm{mg} / \mathrm{kg} /$ day had no influence on the CAT and AChE activities in unstressed rats. However. NSP had a significant and dose-dependent prevention of the increases in the CAT activity in the basal ganglia and hypothalamus of SART-stressed rats: the protecting effects of NSP, at a dose of $100 \mathrm{mg} / \mathrm{kg} /$ day $\times 5$, were $54.4 \%$ in the basal ganglia and $67.6 \%$ in the hypothalamus. NSP also had significant effects in preventing increases in AChE activities in the basal ganglia and hypothalamus, and it prevented a decrease in that in the duodenum.

Figure 9 shows the effect of NSP on the increase in the AChE activity in the duodenum of RWIS rats. The increase was reduced dosedependently to the control level by successive pretreatments with NSP. NSP at a dose of $100 \mathrm{mg} / \mathrm{kg} /$ day $\times 5$ caused $89.4 \%$ protection.

\section{Discussion}

It is interesting that the changes were seen 


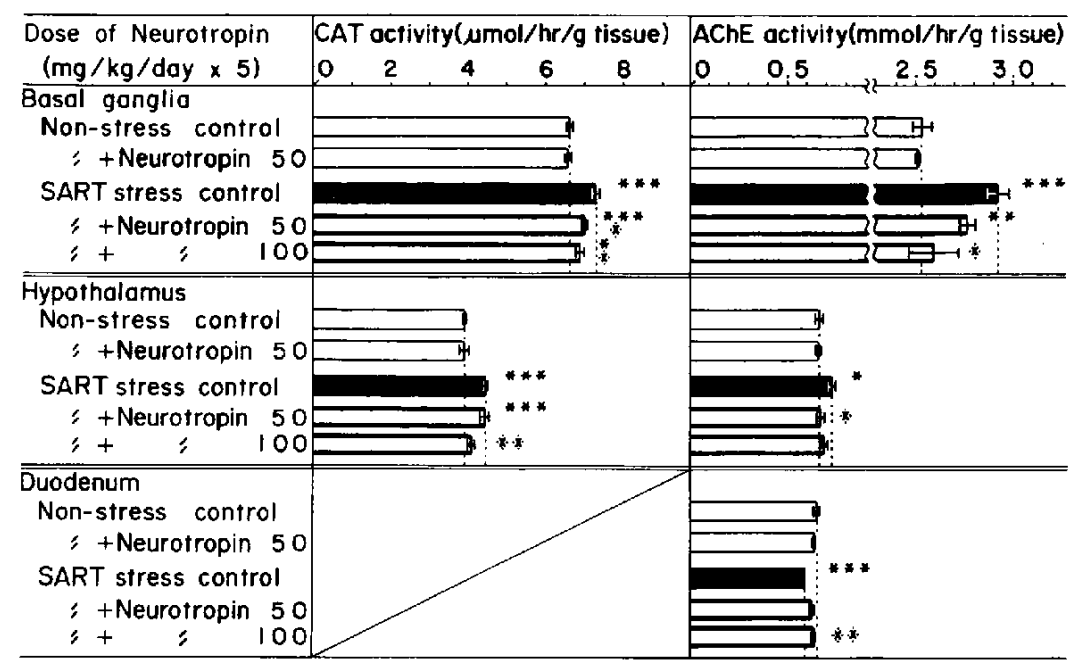

Fig. 8. Effects of Neurotropin on changes of CAT and AChE activities in the brain areas and duodenum of SART-stressed rats. No. of rats: $4-10 /$ group. ${ }^{*} P<0.05,{ }^{* *} P<0.01,{ }^{* * *} P<0.001$ : Significance of difference from the value for non-stressed controls. $\because \mathrm{P}<0.05, \therefore \mathrm{P}<0.01$ : Significance of difference from the value for SART-stressed controls (Student's $t$-test).

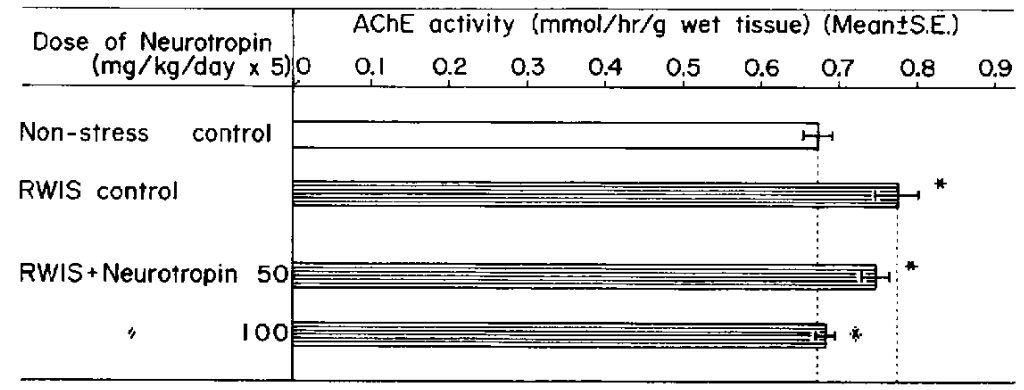

Fig. 9. Effects of Neurotropin on the increase in AChE activity in the duodenum of RWIS rats. No. of rats: 4 or 5 /group. ${ }^{*} P<0.05$ : Significance of difference from the value for the non-stressed controls. : $P<0.05$ : Significance of difference from the value for RWIS controls (Student's $t$-test).

only in the basal ganglia and hypothalamus of the brain of SART-stressed rats in this study and that CAT in the hypothalamus seemed to change most markedly within some parameters since the CAT activity in this area increased markedly from day 1 of SART stress (Fig. 2). The facts that in the basal ganglia and hypothalamus of the brain of cold-stressed rats there were no changes in the CAT or AChE activities and only a slight change in the T-ACh content as seen in Fig. 5 indicate that SART stress is very different from cold stress. Moreover, since no changes in the AChE activities were observed in any area of the brain of RWIS rats, SART stressinduced changes in cholinergic activities are apparently also very different from those induced by common acute stress.

The time courses of changes in the T-ACh content were nearly parallel to those in AChE activities, although there were some differences in changes in different areas (Figs. 1 and 3 ). CAT activity changed most rapidly of the three parameters, especially in the hypothalamus (Figs. 1-3). These results suggest that the change in the CAT activity in the brain may be a trigger for various cholinergic changes in SART-stressed 
animals.

The time course of recovery of the $\mathrm{T}$-ACh content of the duodenum in Fig. 4 after the end of stress is thought to be related to that of the recovery of the number of muscarinic ACh receptors in the small intestine observed in mice by Uchida et al. (15) and the recovery of the response of isolated mouse duodenum to exogenous ACh $(14,28)$.

In SART-stressed rats, the directions of changes in cholinergic parameters in the duodenum as seen in Fig. 6 were considerably different from those in brain areas and were inhibited by bilateral vagotomy at the cardia. These facts show clearly that the changes in the duodenum induced by SART stress are under the control of autonomic centers.

The sedative analgesic NSP has been reported to improve physiological functions of SART-stressed animals (11, 12, 28-31). This study also showed that NSP had protective effects against various cholinergic changes in SART-stressed rats (Figs. 7 and 8). NSP also had a protective effect against change in AChE activity in RWIS rats (Fig. 9). Thus NSP had protective effects in both the partial vagotonic and the sympathicotonic state.

Recently, brain ACh has been studied in relation to mania and depression. Rowntree et al. (32) reported that diisopropylfluorophosphonate caused depression in manics and intensified depression in depressive patients. Janowsky et al. (33) proposed an adrenergic-cholinergic balance hypothesis in relation to $\mathrm{ACh}$ in such disorders; namely. $\mathrm{ACh}$ is dominant over catecholamine in the CNS in depressives and vice versa in manias. Furthermore, they reported that the manic symptoms in manias were decreased transiently by physostigmine and that these decreases were reversed by atropine (34). Thereafter, similar effects of physostigmine were reported by some clinicians (35-39). To prove the hypothesis of $\mathrm{ACh}$ dominance in the relationship between stress and affective disorders, Janowsky and Risch (40) dealt in detail with the roles of the cholinomimetic and anticholinergic drugs in their review.

Recently, cholinergic neurons in the brain have also received attention in relation to Alzheimer's disease, but the important problem of the relevancy of brain cholinergic neurons to this disease has not yet been clarified.

In SART-stressed animals, cholinergic activities were clearly increased in the brain, especially in the hypothalamus, but also in the basal ganglia, judging from measurements of the T-ACh content and the CAT and AChE activities in various areas of the brain.

The above-described facts suggest that abnormalities in the cholinergic activities that are not covered in the so-called stresstheory proposed by Cannon, Selye and others should be reexamined in relation to stress symptoms, dysautonomia, mania, depression, senile dementia (Alzheimer's dementia) and other disorders.

\section{References}

1 Stone, E.A.: Stress and catecholamines. In Catecholamines and Behavior. No. 2, Edited by Friendhoff, A.J., p. 31-72. Plenum Press, New York (1975)

2 Kobayashi, R.M., Palkovits, M., Kizer, J.S., Jacobowits, D.M. and Kopin, I.J.: Selective alterations of catecholamines and tyrosine hydroxylase activity in the hypothalamus following acute and chronic stress. In Catecholamines and Stress, Edited by Usdin, E., Kvetnansky, R. and Kopin, 1.J., p. 29-38, Pergamon Press, Oxford (1976)

3 Saavedra, J.M., Kvetnansky, R. and Kopin, I.J.: Adrenaline, noradrenaline and dopamine levels in specific brain stem areas of acutely immobilized rats. Brain Res. 160, 271-280 (1979)

4 Tanaka, M., Kohno, Y., Nakagawa, R., Ida, Y., Takeda, S. and Nagasaki, N.: Time-related differences in noradrenaline turnover in rat brain regions by stress. Pharmacol. Biochem. Behav. 16. 315-319 (1982)

5 Anisman, H. and Zacharko, R.M.: Depression: The predisposing influence of stress. Behav. Brain Sci. 5, 89-137 (1982)

6 Gilad, G.M., Rabey, J.M. and Shenkman, L.: Strain-dependent and stress-induced changes in rat hippocampal cholinergic system. Brain Res. 267, 171-174 (1984)

7 Costa, E., Tagliamonte, A., Brunello, N. and Cherney, D.L.: Effect of stress on the metabolism of acetylcholine in the cholinergic pathways of extrapyramidal and limbic 
systems. In Catecholamines and Stress: Recent Advances, Edited by Usdin. E., Kvetnansky, R, and Kopin, I.J., p. 48-68, Elsevier. New York (1980)

8 Cherek, D.R., Lane, J.D., Freeman, M.E. and Smith, J.E.: Receptor changes following shock avoidance. Soc. Neurosci. Abstr. 6, 543 (1980)

9 Hingtgen, J.N., Smith, J.E., Shea, P.A., Aprison, M.H. and Gaff, T.M.: Cholinergic changes during conditioned suppression in rats. Science 193 , 332-334 (1976)

10 Kita, T., Hata, T., Yoneda, R. and Okage, T.: Stress state caused by alternation of rhythm in environmental temperature, and the functional disorders in mice and rats. Folia Pharmacol. Japon. 71, 195-210 (1975) (Abs. in English)

11 Kita, T., Hata, T., Itoh, E and Namimatsu, A.: Testing methods for vegetative syndrome in the rat and effects of Neurotropin and other drugs. Japan. J. Psychosom. Med. 23, 61-68 (1983)

12 Hata, T., Kita, T., Namimatsu, A., Itoh, E, and Oda, $Y .:$ Changes of blood pressure and regional blood flow in SART rats and drug actions on these changes. Folia Pharmacol. Japon. 79, 335342 (1982) (Abs. in English)

13 Kita, T., Hata, T., lida, J., Yoneda, R. and Ishida, S.: Decrease in pain threshold in SART stressed mice. Japan. J. Pharmacol. 29, 479-482 (1979)

14 Hata, T., Kita, T., lida, J., Yoshida, H., Uchida, S. and Ishida, S: Decrease of $\mathrm{ACh}$ response in isolated duodenum from repeated cold stressed (SART stressed) mice. J. Pharmacobiodyn. 1, 338-340 (1978)

15 Uchida, S., Takeyasu, K., Noguchi, Y., Yoshida, H., Hata, T. and Kita, T.: Decrease in muscarinic acetylcholine receptors in the small intestine of mice subjected to repeated cold stress. Life Sci. 22, 2197-2204 (1978)

16 Hata, T., lida, J., Kita. T., Itoh, E. and Namimatsu, A.: Influence of vagotomy or sympathectomy on SART stressed animais. Japan. J. NRA 6, 44-45 (1980) (in Japanese)

17 Kita, T., Hata, T., Higashiguchi, T., Itoh, E. and Kawabata, A.: Changes of total acetylcholine content and the activity of related enzymes in SART (repeated cold)-stressed rat brain and duodenum. Japan. J. Pharmacol. 40, 174-177 (1986)

18 Yoneda, R., Kita, T., Hata, T. and Namimatsu, A.: Experimental partial sympathicotonia, and effects of some drugs on it in restraint and water immersion stressed animals. J. Pharmacobiodyn. 3, 692-701 (1980)

19 Yoneda, R., Hata, T., Kita, T., Namimatsu, A. and
Itoh, E.: Experimental pharmacological study on partial sympathicotonia in restraint and water immersion stressed animals. J. Pharmacobiodyn. 4, 251-261 (1981)

20 Hata, T., Kita, T., Itoh, E. and Harada, N.: Experimental studies on optimal conditions of loading SART stress (repeated cold stress) upon animais. Japan. J. Psychosom. Med. 24, 257266 (1984)

21 Gispen, W.H., Schotman, P. and Dekloet, E.R.: Brain RNA and hypophysectomy; A topographical study. Neuroendocrinology 9, 285296 (1972)

22 Toru, M. and Aprison, M.H.: Brain acetylcholine studies: A new extraction procedure. J. Neurochem. 13, 1533-1544 (1966)

23 Yagasaki, O., Kagawa, K., Takewaki, T. and Yanagiya, I.: A method for differential measurement of free and bound acetylcholine in the small intestine. Japan. J. Pharmacol. 22, 425427 (1972)

24 Blaber, L.C. and Cuthbert, A.W.: A sensitive method for the assay of acetylcholine. J. Pharm. Pharmacol. 13, 445-446 (1961)

25 Fonnum, F.: Radiochemical micro assays for the determination of choline acetyltransferase and acetylcholinesterase activities. Biochem. J. 115, 465-472 (1969)

26 Ellman, G.L., Courtney, K.D., Andres, V., Jr. and Featherstone, R.M.: A new and rapid colormetric determination of acetylcholinesterase activity. Biochem. Pharmacol. 7, 88-95 (1961)

27 Toriumi, T., Nakamura, $\mathbf{N}$. and Nagao, F.: Curative effect of a vagotomy on acetic acid ulcer. In Experimental Ulcers, Edited by Umehara, S., Takagi, K., Nagao, F. and Matsuo,

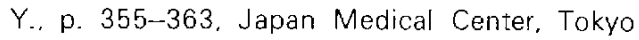
(1976)

28 Kita, T., Hata, T., lida, J. and Ishida, S.: Decrease of $A C h$ response in isolated duodenum from SART stressed (repeated cold stressed) mice. Folia Pharmacol. Japan. 75, 33-44 (1979) (Abs. in English)

29 Kita, T., Hata, T., Okage, T., Yoneda, R. and Hoshino, K.: Effect of Neurotropin upon SARTstress states in mice and rats. Folia Pharmacol. Japon. 71, 211-220 (1975) (Abs. in English)

30 Kita, T., Hata, T. and Yoneda, R.: Analgesic effects of Neurotropin in mice, and comparison between analgesic effects of some drugs in SART-stress mice and normal mice. Folia Pharmacol. Japon. 72, 573-584 (1976) (Abs. in English)

31 Hata, T., Kita, T., Itoh, E. and Namimatsu, A.: Changes of the function in the heart of SART 
stressed (repeated cold stressed) mice and the action of Neurotropin on these changes. Folia Pharmacol. Japon. 79, 487-492 (1982) (Abs. in English)

32 Rowntree, D.W., Neven, S. and Wilson, A.: The effect of disopropylfluorophosphonate in schizophrenia and manic depressive psychosis. J. Neurol. Neurosurg. Psychiatry 13, 47-62 (1950)

33 Janowsky, D.S., El-Yousef, M.K., Davis, J.M. and Sekerke, H.J.: A cholinergic-adrenergic hypothesis of mania and depression. Lancet 1 . 632-635 (1972)

34 Janowsky, D.S., El-Yousef, M.K., Davis, J.M. and Sekerke, H.J.: Parasympathetic suppression of manic symptoms by physostigmine. Arch. Gen. Psychiatry 28, 542-547 (1973)

35 Carroll, B.J., Frazer, A., Schless, A. and Mendels, J.: Cholinergic reversal of manic symptoms. Lancet 1, 427 (1973)
36 Modestin, J.J., Hunger, J. and Schwartz, R.B.: Über die depressogene Wirkung von Physostigmine. Arch. Psychiatr. Nervenkr. 218, 67-77 (1973)

37 Modestin, J.J., Schwartz, R.B. and Hunger, J.: Zur Frage der Beeinflussung schizophrener Symptome Physostigmine. Pharmakopsychiatrie 9, 300-304 (1973)

38 Shopsin, B., Janowsky, D.S., Davis, J.M. and Gershon, S.: Rebound phenomena in mania patients of following physostigmine. Neuropsychobiology 1, 180-187 (1975)

39 Davis, K.L., Berger, P.A., Hollister, L.E. and DeFraites, E.: Physostigmine in man. Arch. Gen. Psychiatry 35, 119-122 (1978)

40 Janowsky, D.S. and Risch, S.C.: Cholinomimetic and anticholinergic drugs used to investigate an acetylcholine hypothesis of affective disorders and stress. Drug Dev. Res. 4, 125-142 (1984) 